Published in final edited form as:

J Child Neurol. 2013 March ; 28(3): 303-307. doi:10.1177/0883073812446310.

\title{
Height Assessments in Children with Neurofibromatosis Type 1
}

\author{
Elizabeth A. Soucy, BS ${ }^{1}$, Dorothy van Oppen, BS ${ }^{1}$, Nicole L. Nejedly, BS ${ }^{2}$, Feng Gao, MD, \\ $\mathbf{P h D}^{3}$, David H. Gutmann, MD, PhD ${ }^{1}$, and Abby S. Hollander, $\mathbf{M D}^{2}$ \\ ${ }^{1}$ Department of Neurology, Washington University School of Medicine, St. Louis, MO 63110
}

${ }^{2}$ Department of Pediatrics, Washington University School of Medicine, St. Louis, MO 63110

${ }^{3}$ Division of Biostatistics, Washington University School of Medicine, St. Louis, MO 63110

\begin{abstract}
Previous studies have suggested that children with neurofibromatosis type 1 (NF1) are shorter than their unaffected counterparts. Unfortunately, these reports did not consider other contributing factors that might also influence short stature. The purpose of the current study was to characterize the genetic influence of NF1 on the growth of children. Height data was measured and recorded for 170 patients, while parental measurements were obtained for 61 patients to calculate sexcorrected mid-parental target heights. Children with NF1 had population mean height and midparental height z-scores statistically different from the general population. Importantly, these differences were pronounced when neither parent had NF1, but were not significant when one of the parents had NF1. Moreover, height z-scores for children with NF1 were also statistically different than their unaffected siblings. Collectively, these data establish a clear effect of a germline NF1 gene mutation on stature in children with NF1.
\end{abstract}

\section{Keywords}

Neurofibromatosis type 1; Stature; Mid-parental target height

Address correspondence to: Abby Hollander, MD, Division of Pediatric Endocrinology and Diabetes, Department of Pediatrics, Box 8116, 660 South Euclid Avenue, St. Louis Children's Hospital and Washington University School of Medicine, St. Louis MO 63110. 314-454-6051 (Phone); 314-454-6225 (FAX); hollander@kids.wustl.edu or David H. Gutmann, MD, PhD, Department of Neurology, Box 8111, 660 South Euclid Avenue, Washington University School of Medicine, St. Louis MO 63110. 314-362-7379 (Phone); 314-362-2388 (FAX); gutmannd@neuro.wustl.edu..

Mailing address for all authors: E.A.S., D.V.O., D.H.G., Department of Neurology, Box 8111, 660 South Euclid Avenue, St. Louis, MO 63110; F.G., Division of Biostatistics, Box 8067, 660 South Euclid Avenue, St. Louis, MO 63110; N.L.N, A.S.H., Department of Pediatrics, Box 8016, 660 South Euclid Avenue, St. Louis, MO 63110

Email addresses for all authors: Elizabeth Soucy - soucye@ neuro.wustl.edu

Dorothy Van Oppen - vanoppend@wusm.wustl.edu

Nicole Nejedly - nejedlyn@wusm.wustl.edu

Feng Gao - feng@wubios.wustl.edu

David Gutmann - gutmannd@neuro.wustl.edu

Abby Hollander - hollander@kids.wustl.edu

AUTHOR CONTRIBUTIONS: EAS, DVO, and NLN collected clinical data.

EAS and FG performed statistical analysis.

EAS, DHG, and ASH prepared the manuscript.

CONFLICT OF INTEREST: The authors declare no potential conflicts of interest with respect to the research, authorship, and/or publication of this article.

ETHICAL APPROVAL: The study protocol was approved by the Human Research Protection Office at the Washington University School of Medicine. 


\section{INTRODUCTION}

Neurofibromatosis type 1 (NF1) is an autosomal dominant inherited tumor predisposition syndrome that affects approximately one in every 3000 individuals worldwide. ${ }^{1}$ In this regard, the defining features of NF1 include tumors of the central and peripheral nervous system (gliomas and neurofibromas). However, children with NF1 are also prone to the development of non-neoplastic features, such as pigmentary abnormalities (café-au-lait spots, inguinal freckling, and Lisch nodules), skeletal anomalies, and short stature. ${ }^{1,2}$ Studies examining short stature in children with NF1 have reported heights two standard deviations or more below the reference population mean in $13 \%$ of affected individuals, ${ }^{3}$ heights that fell below the $3^{\text {rd }}$ centile for the general population in $15 \%$ of children with $\mathrm{NF} 1^{4}$ or heights below the $5^{\text {th }}$ centile in $27-33 \%$ of affected individuals. ${ }^{1,5}$ Although these reports highlight the frequency of short stature in this unique patient population, they fail to account for growth differences between affected children and their parents and unaffected siblings, and do not exclude potential NF1-associated co-morbid etiologies for short stature.

To more accurately characterize the growth patterns that result from a germline mutation in the $N F 1$ gene, we created a database to track growth in children with NF1 lacking obvious risk factors for short stature. We collected height data from parents and siblings so the heights of children with NF1 could be evaluated relative to their individual genetic backgrounds. To our knowledge, this is the first study to compare growth in children with NF1 to their affected and unaffected family members.

\section{METHODS}

An unselected, consecutive series of 231 patients with NF1 were seen at a tertiary care, subspecialty referral Neurofibromatosis Clinic at St. Louis Children's Hospital between 5/19/2010 and 10/27/2011. The diagnosis of NF1 was made according to established diagnostic criteria by a single NF specialist (D.H.G.). ${ }^{2,6}$ The diagnosis of NF1 was made solely by clinical findings using NIH Consensus Development Conference criteria, and no genetic studies were performed.

Patients were recruited to participate in the study if they were over 3 years of age and were able to stand independently for a height measurement. Patients were excluded if they had a known risk for disordered growth: precocious puberty or other endocrine disorder, intracranial tumor, skeletal abnormality, or treatment with attention deficit-hyperactivity disorder (ADHD) medication. In this regard, no patients with skeletal anomalies (scoliosis, pseudarthrosis, long bone deformities, etc.) were included in patients in order to exclude any such effect on patient height. For eligible subjects with NF1, parents were invited to participate in the study unless they had a history of a specific growth disorder in childhood or had a known skeletal disorder. Siblings were invited to participate unless they had a significant medical illness, a known endocrine disorder, or a skeletal abnormality. One hundred and seventy primary subjects agreed to participate in the study ( 94 females and 76 males). Patient demographics, including sex, age, and pertinent clinical information, were also recorded.

Of the 170 patients, parental height information on both parents was measured and recorded for 61 patients and sibling height information was measured and recorded for 19 patients. Sex-corrected mid-parental target height was calculated by taking the arithmetic mean of parental height and adding or subtracting $6.5 \mathrm{~cm}$ for males or females, respectively. ${ }^{7}$ This mid-parental target height was used to estimate the genetically-determined adult height percentile that each patient should obtain if they were unaffected by NF1. Parental and sibling NF1 status (affected or unaffected) was noted. 
Patients' heights, mid-parental target height values, and siblings' heights were standardized to obtain z-scores using the Center for Disease Control (CDC) 2000 growth charts for reference population norms of stature by age and sex. ${ }^{8}$ The z-scores were summarized using means and standard deviations. For patients over 20 years and for the mid-parental target height z-score calculation, we used the z-score corresponding to a 20 year old, which was the upper age limit of the CDC population growth charts. Since the average onset of puberty is 10 years old for girls and 12 years old for boys, patient $\mathrm{z}$-scores for girls 10 years and younger were compared to those over 10 years, and for boys 12 years and younger, they were compared to those over 12 years to assess the effect of NF1 on puberty. ${ }^{9,10}$

One-sample t-tests were used to see whether the average of patients' z-scores (overall and grouped by age) were significantly different from the population level, while paired t-tests were used to compare the average $\mathrm{z}$-scores of patients versus parents (mid-parental target height) as well as patients versus unaffected siblings. All analyses were two-sided and significance was set as $\mathrm{p}$-value $<0.05$. Statistical analyses were performed using the SAS statistical package (SAS Institutes, Cary, NC).

The study protocol was approved by the Human Research Protection Office at the Washington University School of Medicine.

\section{RESULTS}

Heights for children with NF1 were plotted on CDC growth charts (Figures 1 and 2 for boys and girls, respectively) and represented as height z-score data (Table 1). The frequency distribution of standardized z-scores relative to the population mean height revealed that one hundred and sixteen (68\%) children had negative z-scores relative to the population mean height (Figure 3). These affected individuals had a mean height z-score of $-0.50 \pm 1.27$, which was significantly shorter than that observed in the general population $(\mathrm{p}<0.001)$.

The frequency distribution of z-score values relative to mid-parental target height revealed a calculated mid-parental target height $z$-score of $0.06 \pm 0.86$ (Figure 4). The affected patient height $\mathrm{z}$-scores were significantly different from the mid-parental target height $\mathrm{z}$-scores $(\mathrm{p}<0.001)$. This difference was pronounced when neither parent has NF1 ( $<<0.01)$, but was not significant when one of the parents had NF1 $(\mathrm{p}=0.1)$. As predicted, the unaffected sibling $\mathrm{z}$-scores differed from those seen in affected children $(\mathrm{p}=0.01)$, but were not different from the mid-parental target height $\mathrm{z}$-scores $(\mathrm{p}=0.7)$. Height $\mathrm{z}$-scores for girls were not different when those younger than 10 years were compared to those over $10(\mathrm{p}=0.4)$, and for boys, were not different when those younger than 12 years were compared to those over 12 $(\mathrm{p}=0.3)$.

Eight of $61(13 \%)$ patients had height z-scores that differed from the mid-parental target height by 2 standard deviations or more, indicating abnormal growth. Four of these eight individuals (50\%) were within 2 standard deviations of the population mean height. Studies that do not consider genetic targets based on parental height would have classified these children as having normal stature.

\section{DISCUSSION}

Previous studies on short stature in NF1 have reported that 13-33\% of affected individuals are shorter than expected. While these studies highlight this feature in the NF1 pediatric population, they also raise issues about the true incidence of short stature, the impact of a germline NF1 gene mutation on growth, and the contribution of NF1-associated co-morbid 
features. In an effort to address these issues, we used standardized height measurements routinely employed by endocrinologists consulted for the evaluation of short stature.

First, using a combination of population mean height and mid-parental target height assessments, we estimate that true short stature in this affected population, defined as $>2$ standard deviations below the predicted mean, is observed in $8 \%$ of children with NF1. This estimate of population frequency is lower than that reported by $\operatorname{Szudek}^{3}(13 \%)$ and Clementi ${ }^{4}(15 \%)$; however, these studies did not exclude subjects with scoliosis, pseudarthrosis, or other primary causes for short stature. Similar to learning disabilities in children with NF1, ${ }^{11,12}$ the distribution of stature in affected individuals was left-shifted, supporting the conclusion that stature is reduced to some degree in all individuals with NF1, even if they do not have frank short stature.

Second, standardization for age and sex by z-score allowed us to combine measurements across groups that differed in age and sex. Using this method, we showed that short stature reflects the presence of a germline NF1 mutation, in that children with non-familial NF1 (from families with no affected parent) were shorter than their predicted mid-parental target height as well as their unaffected siblings. The fact that this difference between NF1 patients and parents, as assessed by the mid-parental target height z-scores, disappears when one parent has NF1 strongly supports a specific genetic influence of NF1 on growth. This observation is critical to the interpretation of all NF1 height studies, as height is highly heritable in all children, whether or not they have NF1. In this regard, short parents often have short-statured children. ${ }^{13-15}$

While the molecular etiology for short stature in children with NF1 has not been completely elucidated, several studies in $\mathrm{Nfl}$ genetically-engineered mouse strains have revealed a primary effect of $\mathrm{Nfl}$ gene inactivation on bone and somatic growth. In these studies, genetically-engineered mouse strains lacking $N f l$ expression in bone have skeletal abnormalities, ${ }^{16,17}$ while genetically-engineered mouse strains lacking $N f 1$ expression in central nervous system neurons have reduced hypothalamic-pituitary axis function and lower insulin-like growth factor-I (IGF-1) levels. ${ }^{18}$ Future work in both preclinical models and in children with NF1 will be required to determine how these factors contribute to short stature in the pediatric population.

Third, short stature could reflect co-morbid NF1-associated features, such as precocious puberty, ${ }^{19}$ hypothalamic/optic chiasmal gliomas, treatment with stimulant medication, or skeletal abnormalities (pseudarthrosis, scoliosis). ${ }^{20}$ In our series, we specifically excluded individuals with NF1 and short stature due to these causes. Precocious puberty can lead to accelerated bone maturation and result in short stature from premature epiphyseal closure. ${ }^{21,22}$ Our population did not show a difference in height z-scores when we compared prepubertal (girls under 10 years, boys under 12 years) to postpubertal-aged children, so short stature did not appear to be due to early timing of puberty, which would lead to taller stature in early childhood and shorter stature after puberty. Suprasellar lesions, including hypothalamic glioma, may cause growth hormone deficiency (GHD) or precocious puberty. ${ }^{23}$ Attention deficit-hyperactivity disorder (ADHD) is also prevalent in children with NF1 compared to the normal population, and stimulant treatment has been associated with growth deficits. ${ }^{24}$ Finally, growth hormone deficiency in the absence of a suprasellar lesion may be another cause for short stature. ${ }^{5}$

Future studies on individuals with NF1 should focus on the potential mechanisms leading to short stature in this at-risk population. In this regard, characterization of longitudinal growth patterns will be necessary to determine whether pubertal growth is affected more than prepubertal growth. Similarly, an evaluation of circulating growth-related factors (growth 
hormone, IGF-1, and IGF binding proteins) may reveal abnormalities in growth hormone secretion or action. Lastly, measurements of the responses of bone to usual growth mediators may show differences in bone at the cellular level. Collectively, these investigations may further elucidate the causes of NF1-mediated growth dysfunction, and are likely to define the molecular and cellular etiologies for growth failure in children with $\mathrm{NF} 1$ currently classified as having idiopathic short stature.

\section{Acknowledgments}

This study was conducted at the Washington University School of Medicine. We thank Anne C. Albers, MSN, CPNP, Vicki Kuchnicki, BS, and Elaine Cassidy, MS, for their assistance and helpful comments during the execution of this project. The authors wish to acknowledge the support of the Biostatistics Core, Siteman Comprehensive Cancer Center and NCI Cancer Center Support Grant P30 CA091842.

\section{REFERENCES}

1. Friedman, J.; Riccardi, VM. Neurofibromatosis: phenotype, natural history, and pathogenesis. 3rd ed. Johns Hopkins University Press; Baltimore: 1999.

2. Gutmann DH, Aylsworth A, Carey JC, et al. The diagnostic evaluation and multidisciplinary management of neurofibromatosis 1 and neurofibromatosis 2. JAMA. 1997; 278(1):51-57. [PubMed: 9207339]

3. Szudek J, Birch P, Friedman JM. Growth in North American white children with neurofibromatosis 1 (NF1). J. Med. Genet. 2000; 37(12):933-938. [PubMed: 11106357]

4. Clementi M, Milani S, Mammi I, et al. Neurofibromatosis type 1 growth charts. Am. J. Med. Genet. 1999; 87(4):317-323. [PubMed: 10588837]

5. Vassilopoulou-Sellin R, Klein MJ, Slopis JK. Growth hormone deficiency in children with neurofibromatosis type 1 without suprasellar lesions. Pediatr. Neurol. 2000; 22(5):355-358. [PubMed: 10913726]

6. Anon. Neurofibromatosis. Conference statement. National Institutes of Health Consensus Development Conference. Arch. Neurol. 1988; 45(5):575-578. [PubMed: 3128965]

7. Tanner JM, Goldstein H, Whitehouse RH. Standards for children's height at ages 2-9 years allowing for heights of parents. Arch. Dis. Child. 1970; 45(244):755-762. [PubMed: 5491878]

8. Kuczmarski RJ, Ogden CL, Grummer-Strawn LM, et al. CDC growth charts: United States. Adv Data. 2000; (314):1-27. [PubMed: 11183293]

9. Sun SS, Schubert CM, Chumlea WC, et al. National estimates of the timing of sexual maturation and racial differences among US children. Pediatrics. 2002; 110(5):911-919. [PubMed: 12415029]

10. Lee PA. Normal ages of pubertal events among American males and females. J Adolesc Health Care. 1980; 1(1):26-29. [PubMed: 6458588]

11. Eldridge R, Denckla MB, Bien E, et al. Neurofibromatosis type 1 (Recklinghausen's disease). Neurologic and cognitive assessment with sibling controls. Am. J. Dis. Child. 1989; 143(7):833837. [PubMed: 2500844]

12. North KN, Riccardi V, Samango-Sprouse C, et al. Cognitive function and academic performance in neurofibromatosis. 1: consensus statement from the NF1 Cognitive Disorders Task Force. Neurology. 1997; 48(4):1121-1127. [PubMed: 9109916]

13. Macgregor S, Cornes BK, Martin NG, Visscher PM. Bias, precision and heritability of selfreported and clinically measured height in Australian twins. Hum. Genet. 2006; 120(4):571-580. [PubMed: 16933140]

14. Mukhopadhyay N, Finegold DN, Larson MG, et al. A genome-wide scan for loci affecting normal adult height in the Framingham Heart Study. Hum. Hered. 2003; 55(4):191-201. [PubMed: 14566097]

15. Yang J, Benyamin B, McEvoy BP, et al. Common SNPs explain a large proportion of the heritability for human height. Nat. Genet. 2010; 42(7):565-569. [PubMed: 20562875]

16. Zhang W, Rhodes SD, Zhao L, et al. Primary osteopathy of vertebrae in a neurofibromatosis type 1 murine model. Bone. 2011; 48(6):1378-1387. [PubMed: 21439418] 
17. Wang W, Nyman JS, Ono K, et al. Mice lacking Nf1 in osteochondroprogenitor cells display skeletal dysplasia similar to patients with neurofibromatosis type I. Hum. Mol. Genet. 2011; 20(20):3910-3924. [PubMed: 21757497]

18. Hegedus B, Yeh T-H, Lee DY, et al. Neurofibromin regulates somatic growth through the hypothalamic-pituitary axis. Hum. Mol. Genet. 2008; 17(19):2956-2966. [PubMed: 18614544]

19. Zacharin M. Precocious puberty in two children with neurofibromatosis type $I$ in the absence of optic chiasmal glioma. J. Pediatr. 1997; 130(1):155-157. [PubMed: 9003867]

20. Alwan S, Tredwell SJ, Friedman JM. Is osseous dysplasia a primary feature of neurofibromatosis 1 (NF1)? Clin. Genet. 2005; 67(5):378-390. [PubMed: 15811002]

21. Cnossen MH, Stam EN, Cooiman LC, et al. Endocrinologic disorders and optic pathway gliomas in children with neurofibromatosis type 1. Pediatrics. 1997; 100(4):667-670. [PubMed: 9310522]

22. Habiby R, Silverman B, Listernick R, Charrow J. Precocious puberty in children with neurofibromatosis type 1. J. Pediatr. 1995; 126(3):364-367. [PubMed: 7869193]

23. Carmi D, Shohat M, Metzker A, Dickerman Z. Growth, puberty, and endocrine functions in patients with sporadic or familial neurofibromatosis type 1: a longitudinal study. Pediatrics. 1999; 103(6 Pt 1):1257-1262. [PubMed: 10353939]

24. Faraone SV, Biederman J, Morley CP, Spencer TJ. Effect of stimulants on height and weight: a review of the literature. J Am Acad Child Adolesc Psychiatry. 2008; 47(9):994-1009. [PubMed: 18580502] 


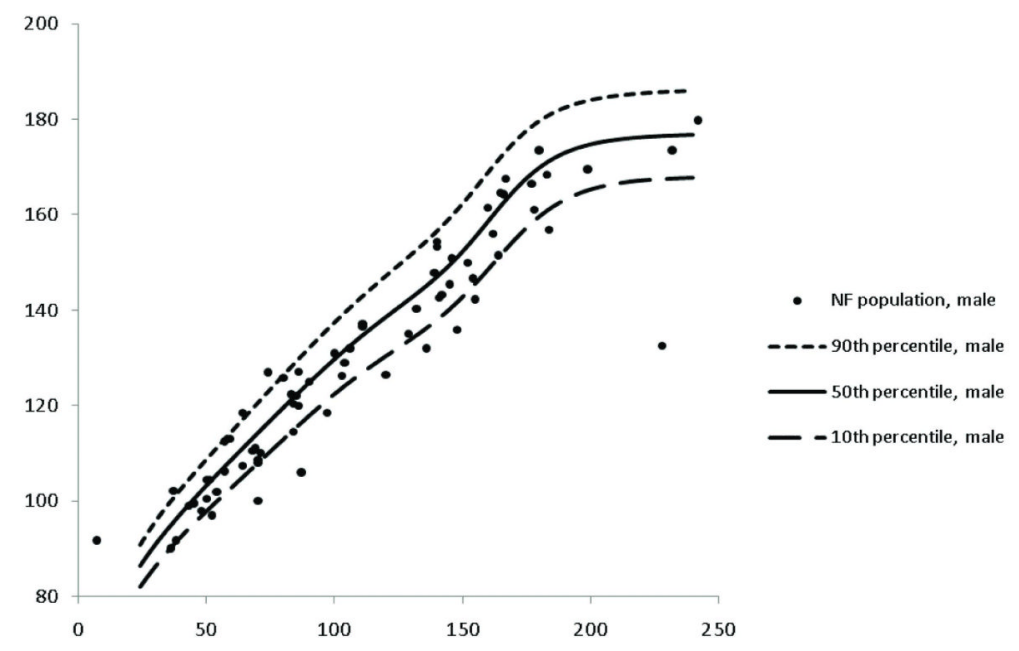

Figure 1. Growth charts of males with NF1

Heights for male children with NF1 are plotted on CDC growth charts to demonstrate distribution of heights. 


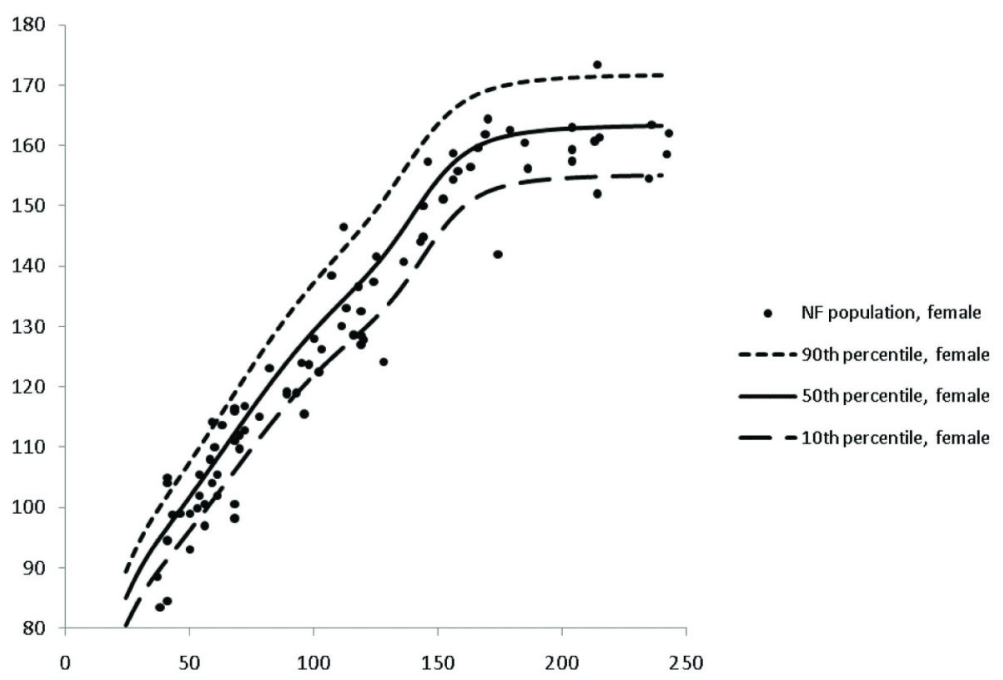

Figure 2. Growth charts of females with NF1

Heights for female children with NF1 are plotted on CDC growth charts to demonstrate distribution of heights. 


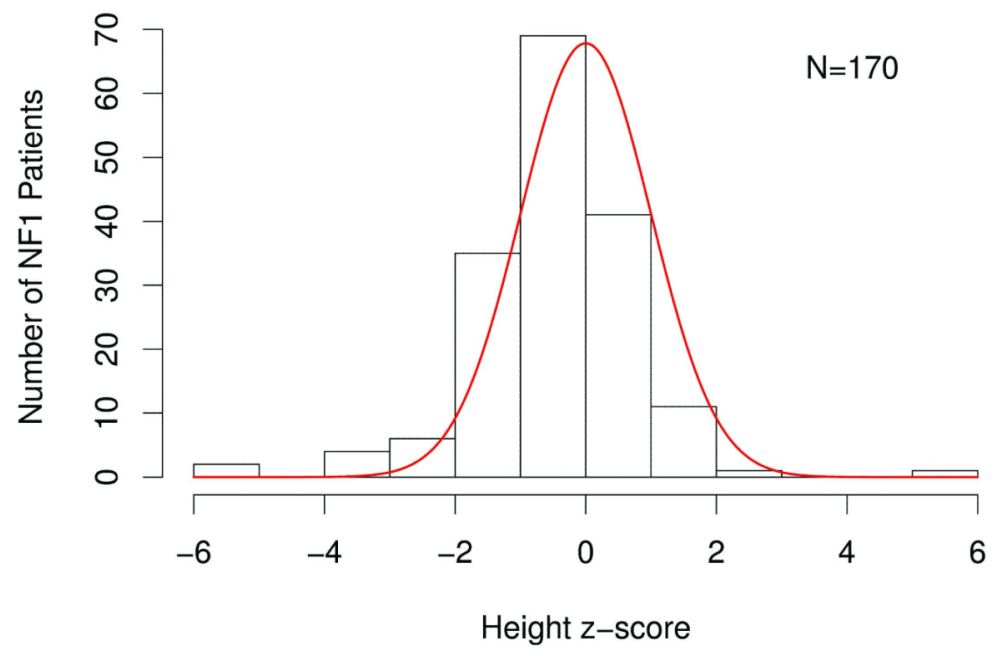

Figure 3. Frequency distribution of standardized $z$-scores in children with NF1

The solid line represents the distribution of mean height $\mathrm{z}$-scores for the general population. Children with NF1 were significantly different from the general population $(\mathrm{p}<0.001)$. 


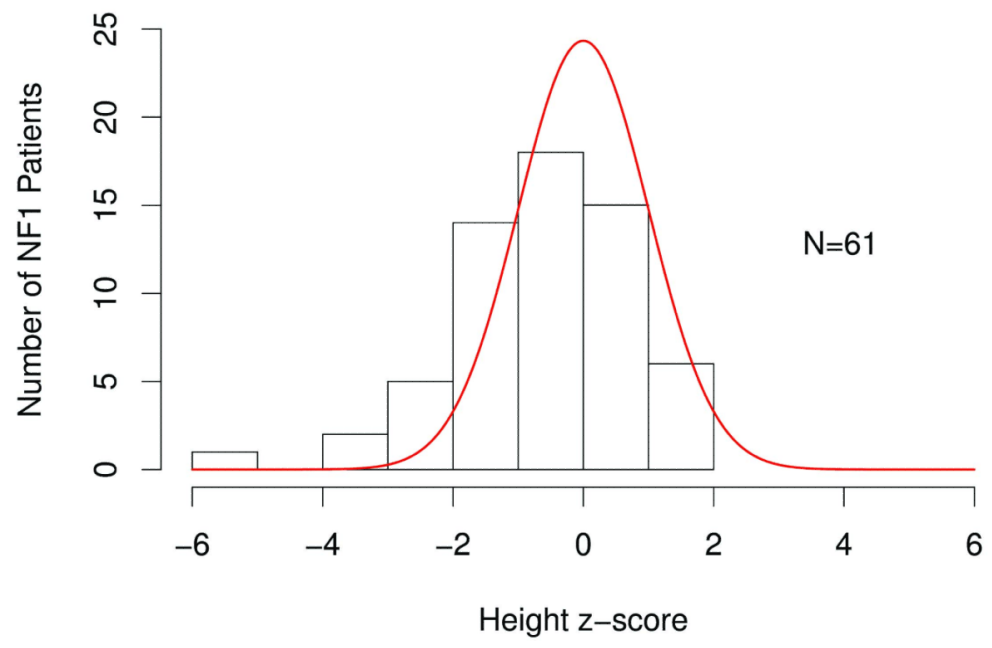

Figure 4. Frequency distribution of $\mathrm{z}$-score values in children with NF1 relative to mid-parental target heights

The solid line represents the distribution of mean height z-scores for the mid-parent target heights. Children with NF1 were significantly different from the predicted mean-parental target heights $(\mathrm{p}<0.001)$. 
Table 1

Mean height z-scores of patients with NF1, parents, and siblings

\begin{tabular}{|l|l|l|l|l|}
\hline Group & $\mathbf{N}$ & Mean \pm SD & Median (range) & $\mathbf{P}^{\#}$ \\
\hline Patients with NF1 & & & & \\
Overall & 170 & $-0.50 \pm 1.27^{*}$ & $-0.53(-6.00-5.98)$ & $<.001$ \\
One parent with NF1 & 40 & $-0.64 \pm 1.70$ & $-0.66(-6.00-5.98)$ & \\
Neither parent with NF1 & 130 & $-0.46 \pm 1.12$ & $-0.49(-5.53-2.01)$ & \\
\hline Mid-parental Target Height (MTH) & & & & \\
Overall & 61 & $0.06 \pm 0.86$ & $0.11(-2.29-1.46)$ & 0.61 \\
One parent with NF1 & 22 & $-0.26 \pm 0.95$ & $-0.41(-2.29-1.05)$ & \\
Neither parent with NF1 & 39 & $0.24 \pm 0.75$ & $0.27(-1.17-1.46)$ & \\
\hline Siblings & 19 & $0.08 \pm 1.05^{* *}$ & $0.31(-2.42-1.81)$ & 0.73 \\
\multicolumn{1}{|l|}{ Overall } & & & & \\
\hline
\end{tabular}

* $\mathrm{p}<.001$ compared to MTH and $\mathrm{p}=0.01$ compared to Siblings

$* *$

not significant $(\mathrm{p}=0.66)$ compared to MTH

\# compared to population mean height $(\mathrm{PMH})$ 